\title{
Influence of incubation in utero on motility and head-to-head agglutination of ejaculated rabbit spermatozoa*
}

\author{
D. V. Brown† and P. L. Senger* \\ Department of Animal Sciences, Washington State University, Pullman, Washington 99164, \\ U.S.A.
}

\begin{abstract}
Summary. Ejaculated spermatozoa were rendered immotile by incubation for $8 \mathrm{~h}$ at $37^{\circ} \mathrm{C}$ in $2.9 \%$ sodium citrate. Immotile spermatozoa $\left(5 \times 10^{6}\right)$ were surgically inseminated into the uterine lumen of does and samples were recovered from the uterus $5,15,30$ and $60 \mathrm{~min}$ after insemination. Incubation in utero led to a resumption of progressive motility and induced head-to-head agglutination. Motility and head-to-head agglutination were highest (64 and $70 \%$ respectively) at $5 \mathrm{~min}$, and declined ( 48 and $49 \%$ ) by $60 \mathrm{~min}$. The percentage of spermatozoa with an intact acrosome did not change during the in-utero incubation. When spermatozoa from a single ejaculate were evaluated in different females there was significant variation $(P<0.01)$ in the reinitiation of motility and agglutination. Most agglutinated spermatozoa $(>96 \%)$ had an intact acrosomal membrane while acrosomal integrity of single spermatozoa differed greatly among females. We conclude that the agglutinated (motile with intact acrosomes) and non-agglutinated (usually immotile and with disrupted acrosomes) represent different populations within the uterine lumen.
\end{abstract}

\section{Introduction}

Determination of the percentage of progressively motile spermatozoa is still the most widely used criterion to assess seminal quality. The assumption is made that ejaculates with low sperm motility immediately after collection will result in low fertility and that the decrease in motility normally associated with dilution and in-vitro incubation is irreversible. Brown \& Senger (1980) found that heat-treated heifer serum reinitiated progressive motility of bovine spermatozoa previously rendered immotile by in-vitro incubation for $8 \mathrm{~h}$ in $2.9 \%$ sodium citrate. Ejaculates with low sperm motility immediately after collection responded to serum as well as or better than ejaculates with high sperm motility after collection. Brown \& Senger (1980) suggested that motility estimates made immediately after ejaculation do not allow prediction of the potential for motility after serum addition. Uterine fluid is similar in composition to blood serum (Beier, 1974; Menezo, 1975); therefore, we tested the hypothesis that uterine incubation could reverse the decrease in motility of spermatozoa normally associated with dilution and in-vitro incubation.

Brown \& Senger (1980) also found that spermatozoa that became motile after addition of

* Present address and reprint requests: Dr P. L. Senger, Dairy Breeding Research Center, The Pennsylvania State University, University Park, Pennsylvania 16802, U.S.A.

$\dagger$ Present address: Department of Physiology and Biophysics, Colorado State University, Fort Collins, Colorado 80523, U.S.A. 
serum tended to agglutinate. Agglutination has usually been regarded as an artefact or a physiological phenomenon related to cellular immobilization and infertility (Mann, 1964), even though serum-induced agglutination of spermatozoa requires an intact acrosomal membrane (Senger \& Saacke, 1976; Aalseth, Senger \& Becker, 1978; Brown \& Senger, 1980) and vigorous motility (Aalseth et al., 1978; Brown \& Senger, 1980). The second part of this study evaluated the variation in motility, acrosomal integrity and agglutination of spermatozoa from a single ejaculate when inseminated into different females.

\section{Materials and Methods}

\section{Experiment 1}

Each of 9 ejaculates, collected from 1 of 2 New Zealand White rabbits, was diluted to $50 \times$ $10^{6}$ spermatozoa $/ \mathrm{ml}$ in $2.9 \%(\mathrm{w} / \mathrm{v})$ sodium citrate dihydrate $(\mathrm{pH} 7.0,300$ mosmol) containing $500 \mu \mathrm{g}$ potassium penicillin $\mathrm{G} / \mathrm{ml}$ and $50 \mu \mathrm{g}$ dihydrostreptomycin $/ \mathrm{ml}$. Preliminary studies showed that rabbit spermatozoa incubated for $8 \mathrm{~h}$ at $37^{\circ} \mathrm{C}$ in $2.9 \%$ sodium citrate became immotile with only a $5 \%$ decrease in acrosomal integrity. Therefore, all ejaculates were incubated in $2.9 \%$ sodium citrate for $8 \mathrm{~h}$ at $37^{\circ} \mathrm{C}$. To monitor changes in percentage of motile spermatozoa, percentage of intact acrosomes, and percentage of head-to-head agglutination, aliquots were evaluated from each sample at $1 \mathrm{~min}, 2,4,6$ and $8 \mathrm{~h}$. Motility was estimated at $37^{\circ} \mathrm{C}$ using a phase-contrast $(\times 160)$ microscope, and the percentages of spermatozoa with intact acrosomes (Saacke \& White, 1972) and head-to-head agglutination (Senger \& Saacke, 1976) were assessed from fixed samples (Johnson, Berndtson \& Pickett, 1976) using a differential interference contrast $(\times 1000)$ microscope.

After the $8 \mathrm{~h}$ in-vitro incubation, $0.1 \mathrm{ml}$ of the sperm suspension was inseminated surgically into each uterine horn of 36 adult does. Does were anaesthetized with urethane (Sigma) and the cervices were exposed by midventral abdominal incision and an incision through the vaginal wall. Insemination was accomplished by inserting a $20-\mu$, fire-polished, capillary pipette through the external cervical os and into the uterine lumen. The sperm suspension was slowly injected into the uterine lumen from a $1-\mathrm{ml}$ syringe. Insemination equipment was kept at $37^{\circ} \mathrm{C}$. After insemination, the reproductive tract was returned to the abdominal cavity and the midventral incision was temporarily closed until the time of sperm recovery. Manipulation of the female reproductive tract was kept to a minimum. Heat lamps were used to maintain all organs and equipment at $37^{\circ} \mathrm{C}$ during insemination and recovery. Spermatozoa were recovered after 5,15 , 30 and $60 \mathrm{~min}$ by inserting a fire-polished $20-\mu \mathrm{l}$ capillary pipette through the cervix and aspirating uterine fluid by capillary action. To prevent exposure of the recovery pipette to cervical mucus, a larger pipette with one end sealed by parafilm was first inserted through the internal cervical os and the recovery pipette was then inserted through the seal and into the uterine lumina. The capillary pipette was manipulated through the length of the uterine lumen and $20-40 \mu \mathrm{l}$ were recovered. Only one sample was recovered from each uterine horn throughout the study.

An aliquot of each sample was examined for percentage of motile spermatozoa. The estimate of motility was highly subjective in samples with a high degree of agglutination. A second aliquot of the uterine sample was mixed on a microscope slide with an equal volume of $0.2 \%$ glutaraldehyde; the percentages of spermatozoa with intact acrosomes and head-to-head agglutination were determined as described above.

Uterine samples in which erythrocytes were microscopically visible or from does that were not in the follicular phase, as shown by ovarian examination, were excluded from the results. Although 36 does were inseminated, only 12 uterine samples for each of the four incubation times were included in the statistical analysis. Data were analysed using a nested analysis of variance testing main effects: male $($ d.f. $=1)$, ejaculates within male $($ d.f. $=7)$, does within 
ejaculates $($ d.f. $=16)$ and time within doe (d.f. $=23$ ). Variation among times in utero was analysed using one-way analysis of variance, and, when $P<0.05$, differences among means were compared using Duncan's new multiple range test.

\section{Experiment 2}

In Exp. 1, variation in motility and agglutination was observed between females; therefore, variation attributable to females was studied in detail. For each of 4 ejaculates from 1 buck, 12 does were inseminated with semen incubated in vitro as described above. One uterine sample was recovered from each uterine horn after $5 \mathrm{~min}$ in utero. An aliquot was immediately evaluated at $37^{\circ} \mathrm{C}$ for percentage motility. A second aliquot was fixed and evaluated $2-10 \mathrm{~h}$ later for percentage of spermatozoa which were agglutinated, percentage of agglutinated spermatozoa with intact acrosomes and percentage of non-agglutinated spermatozoa with intact acrosomes.

There was no significant difference due to uteri within does; therefore, data from 45 of the 48 females were analysed using a nested analysis of variance testing main effects: ejaculate (d.f. $=$ 3 ), does within ejaculates (d.f. $=41$ ), and horn within does (d.f. $=40$ ). Data from 3 does were excluded due to erythrocyte contamination.

\section{Results}

\section{Experiment 1}

All spermatozoa were immotile before uterine insemination (Text-fig. 1a) and the percentage of spermatozoa with intact acrosomes had declined only slightly during the in-vitro incubation (Text-fig. 1b). After uterine insemination, motility and agglutination were highest at $5 \mathrm{~min}$ in utero and declined $(P<0.01)$ by $60 \mathrm{~min}$. The decline in motility and agglutination was correlated $(r=0.60, P<0.01)$. Acrosomal integrity did not change $(P>0.01)$ during the

(a) Motility

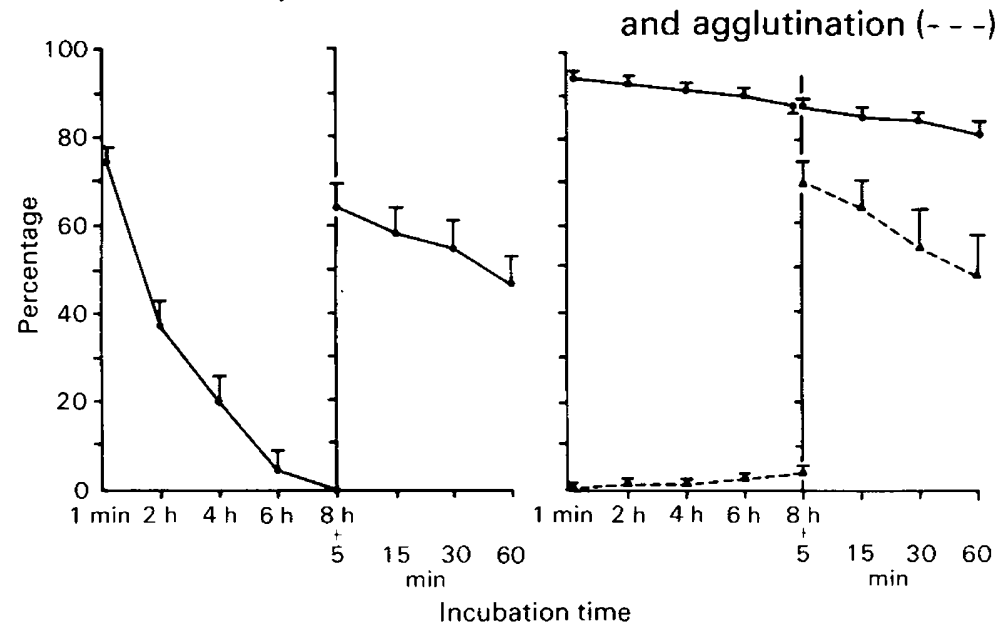

Text-fig. 1. Effect of incubation at $37^{\circ} \mathrm{C}$ in $2.9 \%$ sodium citrate followed by in-utero incubation on (a) percentage of motile spermatozoa and (b) on percentage of intact acrosomes $(-)$ and head-to-head agglutination (---). Each point is the mean ( \pm s.e.m.) for 9 ejaculates in vitro or samples from 12 uterine horns from different females. 
in-utero incubation and was consistently higher than agglutination for each in-utero evaluation (Text-fig. 1b). Nevertheless, agglutination was positively correlated with acrosomal integrity $(r=$ $0.49 ; P<0.01)$.

Motility and acrosomal integrity varied among ejaculates within males $(P<0.05)$ but variation in agglutination among ejaculates, was not significant $(P>0.05)$ because of the large amount of variation associated with females.

\section{Experiment 2}

Female rabbits differed $(P<0.01)$ in their ability to induce progressive motility, agglutination of spermatozoa and the maintenance of acrosomal integrity associated with the non-agglutinated spermatozoa (Text-fig. 2). The percentage of motile spermatozoa and

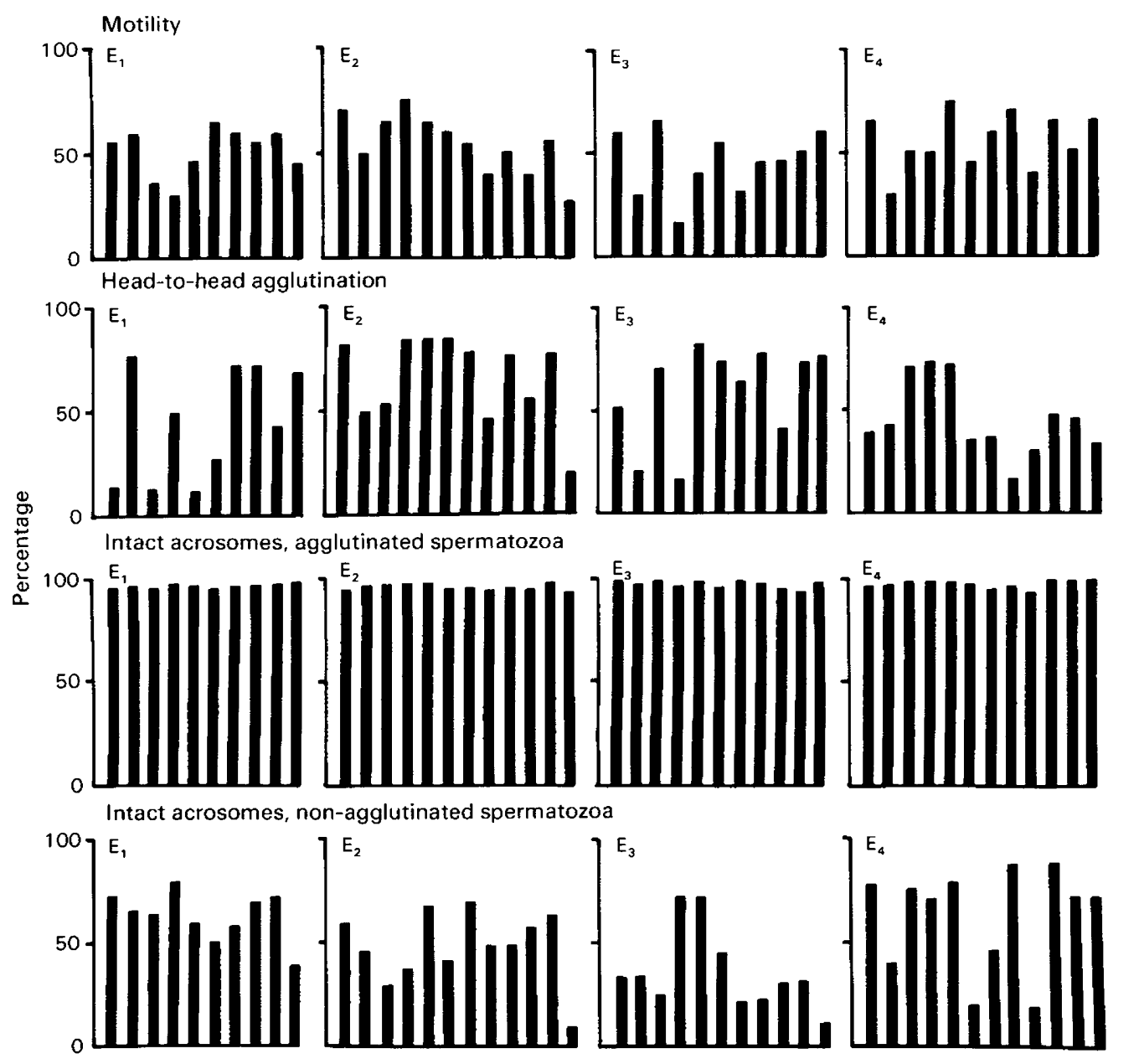

Text-fig. 2. The influence of $5 \mathrm{~min}$ incubation in utero on percentage motility, head-to-head agglutination, intact acrosomes of agglutinated spermatozoa, and intact acrosomes of single spermatozoa. Aliquots from one ejaculate (E) were used to inseminate each group of females. Four ejaculates $\left(E_{1}-E_{4}\right)$ were used, for a total of 45 females inseminated. Each bar represents the mean result for the left and right uterine horn of individual females. 
PLATE 1

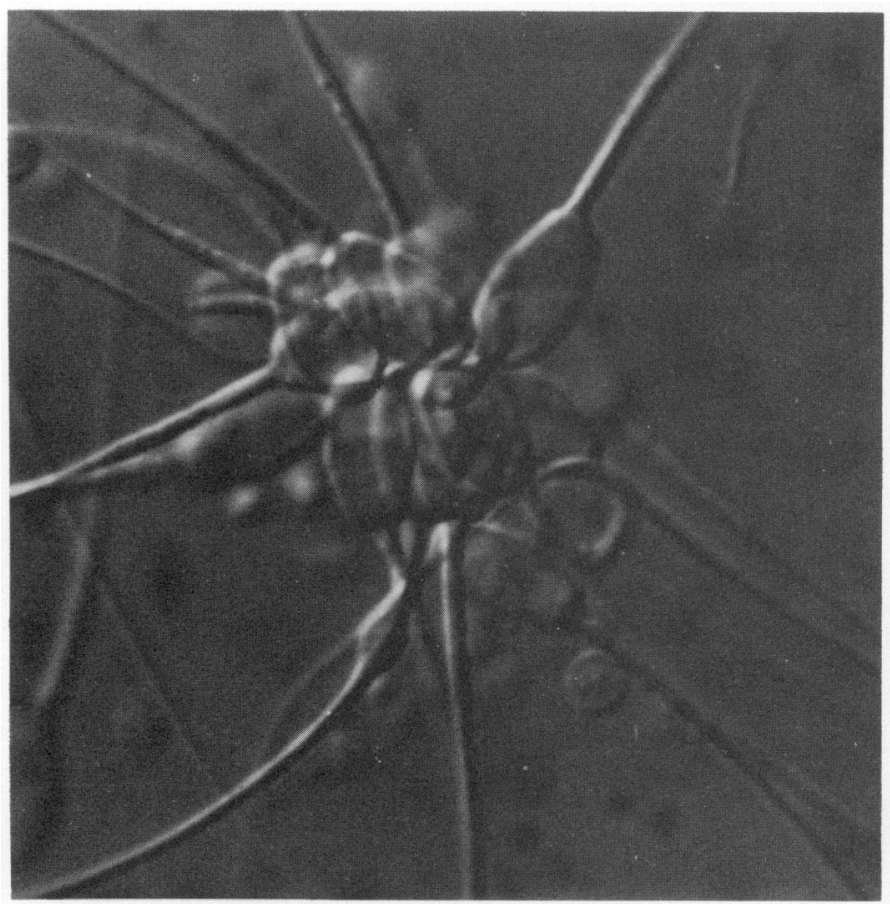

Differential interference-contrast micrograph, illustrating the predominant radial orientation of spermatozoa agglutinated after $5 \mathrm{~min}$ incubation in utero. $\times 5000$.

(Facing p. 286) 
agglutinated spermatozoa ranged from 15 to $75 \%$ and 17 to $79 \%$, respectively. The correlation between motility and agglutination of spermatozoa incubated for 4 min in utero was significant but low $(r=0.34 ; P<0.01)$.

The acrosomal integrity of the agglutinated spermatozoa was consistently high $(>96 \%)$ in all 45 females and little variation among females was observed (Text-fig. 2). Conversely, a large degree of variation $(P<0.01)$ was observed in the acrosomal integrity of the non-agglutinated spermatozoa among females (Text-fig. 2). When the variation associated with females was removed, ejaculates differed in agglutination $(P<0.05)$ and the acrosomal integrity of single spermatozoa $(P<0.05)$ and after $5 \mathrm{~min}$ in utero.

\section{Morphological observations}

The number of spermatozoa per group of agglutinated cells ranged from 2 to $>30$ and averaged approximately 12 spermatozoa (Plate 1). The radial orientation shown is typical. Vigorous motion of the flagellum was a characteristic of agglutinated spermatozoa at all evaluations. In contrast, most non-agglutinated spermatozoa were immotile or vibrated without moving.

\section{Discussion}

Exposure of immotile spermatozoa to the uterine environment resulted in reinitiation of progressive motility of rabbit spermatozoa. Stimulation of sperm respiration following in-utero incubation in the rabbit is well documented (Hamner \& Williams, 1963; Mounib \& Chang, 1964; Iritani, Gomes \& VanDemark, 1969); however, the present study demonstrated that loss of motility due to dilution and incubation for $8 \mathrm{~h}$ in sodium citrate is reversible, and reinitiation of motility occurs immediately $(5 \mathrm{~min})$ after in-utero incubation. The biochemical basis for the loss of motility following $8 \mathrm{~h}$ incubation in sodium citrate is not known, nor has the identity of the motility stimulating substance in uterine fluid been determined. Uterine fluid and blood serum contain pyruvate and lactate (Bavister \& Yanagimachi, 1977), but previous studies (Brown \& Senger, 1980) determined that serum-induced reinitiation of motility was not mediated by the replacement of an oxidizable energy source or divalent ions. Overstreet \& Cooper $(1975,1978$, 1979) reported that over $70 \%$ of rabbit spermatozoa recovered from the uterus showed vigorous progressive motility. They used a flushing medium containing $5 \%$ heat-treated serum which has been shown to stimulate motility and cause agglutination (Brown \& Senger, 1980). The method for removing spermatozoa by capillary action used in this study prevented subjection of spermatozoa to a recovery medium, dilution, or the mechanical effects of flushing. Our technique, however, was restricted to sampling only luminal spermatozoa and no account could be taken of spermatozoa which might have been attached to the endometrium or present in the uterine glands. Only one sample could be recovered from each uterine horn due to the increased incidence of erythrocyte contamination. The observation that motility and agglutination were highest at $5 \mathrm{~min}$ may be due to an inability to sample spermatozoa from the endometrial folds or it could be the result of selective transport of agglutinated spermatozoa. The dynamics of sperm transport could not be evaluated using our recovery technique. However, the fact that in all females spermatozoa again became progressively motile after in-utero incubation clearly shows that the uterine environment is capable of reversing the loss of motility normally associated with dilution and in-vitro incubation.

The fact that head-to-head agglutination occurs in utero is often ignored in studies involving sperm physiology. Mann (1964) summarized the information on agglutination of spermatozoa and reported that this phenomenon was the result of either (1) non-specific in vitro treatments 
such as dilution, washing, cold-shock, changes in $\mathrm{pH}$ and osmotic pressure; or (2) a specific immunological reaction. Consequently, the incidence of agglutination is often regarded as an artefact. This study shows that agglutination is a physiological phenomenon which occurs in the uterus independently of the medium used for sperm recovery.

Single and agglutinated spermatozoa apparently represent distinctly different populations within the uterine lumen: the agglutinated spermatozoa are vigorously motile and have intact acrosomal membranes. Cohen \& Tyler (1980) have also proposed that there are two distinct populations of spermatozoa in the rabbit uterus based on immunofluorescence microscopy. They proposed that a small number of spermatozoa which do not bind IgG are refractory to phagocytosis and are capable of fertilization. The coating of spermatozoa by $\operatorname{IgG}$ or other immunoglobulins may relate to the incidence of agglutination.

The observation that $>96 \%$ of agglutinated spermatozoa in all females had intact acrosomes and vigorous motility while the single spermatozoa showed considerable variation in acrosomal integrity (10-87\%) and motility (15-17\%) indicates that head-to-head agglutination in utero is a selective process dependent on membrane integrity and motility. Senger \& Saacke (1976) reported that bovine spermatozoa agglutinated after addition of serum had a high percentage of intact acrosomes. A positive correlation between serum-induced agglutination and acrosomal integrity (Senger \& Saacke, 1976; Aalseth et al., 1978; Brown \& Senger, 1980) and motility (Aalseth et al., 1978, Brown \& Senger, 1980) is well documented for bovine spermatozoa. The correlations between agglutination and acrosomal integrity and motility were confirmed for in-utero agglutination of rabbit spermatozoa. Brown \& Senger (1980) suggested that vigorous motility is important to the process of agglutination by increasing the probability of cellular association such that maximum agglutination can occur. Head-to-head agglutination may be a good indicator of sperm quality since it incorporates the characteristics of motility and acrosomal integrity.

The physiological function of head-to-head agglutination remains unknown. The fact that agglutination occurs in the uterus after surgical insemination and natural mating suggests a wide range of functional possibilities. Head-to-head agglutination is a reversible process (Senger \& Saacke, 1976; Brown \& Senger, 1980) and many of the spermatozoa dispersed after agglutination are motile and capable of penetration of the ovum (Yanagimachi, 1970). The selective nature of head-to-head agglutination and its relation to acrosomal integrity and motility indicates that agglutination may function in selective sperm transport, to preserve membrane integrity by a resistance to phagocytosis by uterine leukocytes, or to prevent premature capacitation and the acrosome reaction.

We thank W. C. Becker and J. F. McCutchan for assistance and Mr Craig Marvin for the natural mating studies. This is Scientific Paper No. 5779 of the College of Agriculture Research Center, Washington State University, Project 0237.

\section{References}

Aalseth, E.P., Senger, P.L. \& Becker, W.C. (1978) The relationship of sperm viability and concentration to serum-induced head-to-head agglutination of bovine spermatozoa. J. Reprod. Fert. 53, 193-196.

Bavister, B.D. \& Yanagimachi, R. (1977) The effects of sperm extracts and energy sources on the motility and acrosome reaction of hamster spermatozoa in vitro. Biol. Reprod. 16, 228-237.

Beier, H.M. (1974) Oviducal and uterine fluids. $J$. Reprod. Fert. 37, 221-237.
Brown, D.V. \& Senger, P.L. (1980) Influence of homologous blood serum on motility and headto-head agglutination in nonmotile ejaculated bovine spermatozoa. Biol. Reprod. 23, 271-275.

Cohen, J. \& Tyler, K.R. (1980) Sperm populations in the female genital tract of the rabbit. J. Reprod. Fert. 60, 213-218.

Hamner, C.E. \& Williams, W.L. (1963) Effect of female reproductive tract on sperm metabolism in the rabbit and fowl. J. Reprod. Fert. 5, 143-150. 
Iritani, A., Gomes, W.R. \& VanDemark, N.L. (1969) The effect of whole, dialyzed and heated female genital tract fluids on respiration of rabbit and ram spermatozoa. Biol. Reprod. 1, 77-82.

Johnson, L., Berndtson, W.E. \& Pickett, B.W. (1976) An improved method for evaluating acrosomes of bovine spermatozoa. J. Anim. Sci. 42, 951-954.

Mann, T. (1964) The Biochemistry of Semen and of the Male Reproductive Tract, pp. 339-365. Methuen, London.

Menezo, Y.J.R. (1975) Amino constituents of tubal and uterine fluids of the estrous ewe: comparison with blood serum and ram seminal fluid. In The Biology of Spermatozoa, pp. 174-181. Eds E. S. E. Hafez \& C. G. Thibault. Karger, Basel.

Mounib, M.S. \& Chang, M.C. (1964) Effect of in utero incubation on the metabolism of rabbit spermatozoa. Nature, Lond. 201, 943-944.

Overstreet, J.W. \& Cooper, G.W. (1975) Reduced sperm motility in the isthmus of the rabbit oviduct. Nature, Lond. 258, 718-719.
Overstreet, J.W. \& Cooper, G.W. (1978) Sperm transport in the reproductive tract of the female rabbit. I. The rapid transit phase of transport. Biol. Reprod. 19, 101-114.

Overstreet, J.W. \& Cooper, G.W. (1979) Effect of ovulation and sperm motility on the migration of rabbit spermatozoa to the site of fertilization. $J$. Reprod. Fert. 55, 53-59.

Saacke, R.G. \& White, J.M. (1972) Semen quality tests and their relationship to fertility. Proc. 4th Tech. Conf. A.I. \& Reprod., pp. 22-27.

Senger, P.L. \& Saacke, R.G. (1976) Serum-induced head-to-head agglutination of bovine spermatozoa. $J$. Reprod. Fert. 47, 215-219.

Yanagimachi, R. (1970) In vivo capacitation of golden hamster spermatozoa by homologous and heterologous blood sera. Biol. Reprod. 3, 147-153.

Received 21 December 1981 